\title{
Families and Friends group: a group that straddles teaching, support and
}

\section{$\underline{\text { transformation }}$}

Michelle Radcliffe, Jale Cilasun

\begin{abstract}
This paper describes the structure, process and evaluation of a Families and Friends Group in a Personality Disorder Treatment service for people experiencing borderline personality disorder using a Mentalization Based Therapy model. We highlight the benefits of working with family members and consider the differences between psycho-education, support groups and group-analytical approaches. Inclusion of families in recovery is well argued. Providing support and education for family members can improve communication and reduce burden in families. In the treatment of patients with borderline personality disorder, the focus often remains upon meeting patients' needs and not of those who support them. We used a formal evaluation of the effectiveness of the Families and Friends Group in the areas of learning and support. Qualitative questions were used to gain more in depth information regarding the impact of the group.
\end{abstract}

Our results support the approach of working with the family rather than only focusing on the individual. There was an improvement in the communication leading to positive change in relationships between family members and their loved ones. This 
takes place despite the group being a once a month and drop-in. However as this was evaluation of one service, more research in this area is needed.

Keywords; borderline personality disorder, family support groups, Mentalisation Based Therapy, Recovery

\section{Introduction}

This paper describes a families and friends group attached to a Personality Disorder intensive treatment service within an NHS trust which uses a Mentalization Based Therapy (MBT) framework of weekly individual and group therapy. Mentalization is the imaginative mental activity that enables us to perceive and interpret human behaviour in terms of intentional mental states (e.g. needs, desires, feelings, beliefs, goals, purposes and reasons). It is a process and not a given but a developmental achievement (Bateman and Fonagy, 2012). Poor mentalizing can have a devastating effect on the ability to develop and maintain relationships and manage emotions. Therefore supporting family members to understand the impact of poor mentalizing is key to improving communication and relationships. Mentalization Based Therapy was developed by Bateman and Fonagy (2006) and is one of the recommended evidence based treatments for Borderline Personality Disorder (BPD) in the NICE guidelines (NICE, 2009). 
Our aim in writing this paper is to bring our experience into the public arena with the hope that it may stimulate discussion and inspire more services to offer family groups. The group takes place out of hours in the evening once a month. As a service we are not contractually obligated to provide a group for family members, and furthermore some of the families attending do not have a family member currently in our or any other mental health service. Thus we the authors of this paper hold the group due to our personal conviction and commitment to involving and supporting families in improving health, having clinically observed a whole family approach to be much more effective than treating the individual only.

\section{Background}

The impact of caring for a loved one significantly affects family relationships, the carers' physical and mental health as well as putting stress on financial and social resources of the family (Rethink, 2003). The personality disorder capabilities framework in the UK recommended the development of dedicated personality disorder services and the creation of a mental health workforce with a better understanding of personality disorder as well as its impact on individuals, families and society. The framework recommends that clinicians contribute to positive partnerships with patients and their carers. Although as such there is a national recommendation supporting psychosocial family interventions, there is little published work which demonstrates the involvement of families in treatment in personality disorder services. A notable exception is Porr's work (2010), who 
advocates for inclusion of families in the treatment of Borderline Personality Disorder. She argues that failing to involve families is a major reason for clients dropping out of therapy and states that families will be there for the person when clinicians are no longer involved. Furthermore Porr (2010) suggests specific training for family members which enables them to manage crises more effectively and to help develop insight into their relationships.

Hoffman, Fruzzetti and Buteau (2007) argued that family members are perhaps the most affected and in turn family members are most likely to affect the individual with the disorder as personality disorders occur in the context of relationships. They state that in the area of personality disorder, family work with carers is far behind compared to axis I disorders such as Schizophrenia. In the U.K., the National Institute for Clinical Excellence (NICE) for Borderline Personality Disorder recommend involving families and carers in treatment and providing information regarding local support groups where they exist (NICE, 2009). Additionally NHS England have recommended that Clinical Commissioning Groups improve the quality of life of people living with long term and complex health conditions by providing support for carers. As articulated by Weegmann and Head's (2016) article, offering support and therapy for families and carers of people with a diagnosis of personality disorder reflects the socio-political situation in the UK where carers' needs have equal footing with the patients. This is the context in which this Families and Friends Group was developed. 


\section{Description of the Personality Disorder Treatment Service}

The service uses the MBT model for treatment of Borderline Personality Disorder. Patients are referred by NHS Community Mental Health Teams or other specialist services such as eating disorders services within a London Borough. The service is for adults of working age from ages 18 and above. Although there is no older age limit, in practice the oldest patient in the service has been 70, with the mean age being 37.The patients are in majority female, the last 4 years the gender distribution being $73 \%$ female to $27 \%$ male.

There are 48 places in the treatment program. After a 12 week introductory group introducing the basic concepts of MBT and specific skill building exercises, the patients enter the 18 month program which consists of one individual and one group session per week. After the 18 months, there is a six month step-down period during which the treatment intensity is reduced. Patients are made aware of the Families and Friends Group when they join the service and are given the option of asking their loved one if they would like to attend.

\section{Rationale for the Families and Friends Group}

Members of families of people diagnosed with borderline personality disorder are prone to particular stresses and difficulties as outlined above (Rethink, 2003; Hoffman et al.,2007) However despite this and the recommendations for involving carers listed above, family members are not easily included in borderline personality 
disorder services, and there is little published on the types of interventions in use, with the Weegman and Head paper a notable exception (2016). It made common sense to us to offer support to family members, as they frequently have to manage the emotional crises at home. At the time the service was being formed in 2012, we were also approached by two different carers if we had a group available as in the neighbouring borough.

We started with the question, 'How might we offer something of use to families and carers of the patients we treat?'. We sought information and visited other services which have had families' groups. Providing information on the treatment and diagnosis seemed to be helpful, as was the peer support element of sharing difficulties. We hypothesised that family members might also function as a type of auxillary therapists at home, especially if the same language of therapy is used with the patients and their families. Thus this would be not only support for the family members, but in turn might support the patients' treatment too. We started with a group that had a similar structure to the MBT Introductory group, beginning with education about the condition and the problems with mentalizing. We left the structure loose enough to incorporate what the family members found useful. Over time the structure developed which we describe below. 


\section{Structure and Process of the Families and Friends Group}

The Families and Friends Group is available during the time the patient is engaged in the treatment or in the step down phase, and after discharge. However some Families and Friends Group members have joined the group after hearing about it from other group members.

The members are invited by the patients of the service, who give the information about the group to their family members whom they would like to invite. An SMS text is sent in the week before the group is due to meet, as a reminder. The group meets once a month in the evening for 90 minutes. Information about the group is given to the patients of the service during the preparatory phase and repeatedly during clinical meetings and reviews that follow in their treatment journey. The group has been running for over three years with a core membership of between 6-8 group members mostly consisting of parents and some partners or spouses. Over this time a total of 18 people have attended, in a drop-in manner with people attending as and when they want to. Some have attended only once, some have come regularly since the beginning and some use it for a while and stop.

We introduce the Families and Friends Group with the function of providing information about the diagnosis, the treatment approach, the concept of mentalization and of developing skills, as well as peer support for each other. The group’s boundaries are that any information shared remains in the group and we maintain 
confidentiality between this group and the patients' treatment. This causes a particular problem for us, the staff members of this group, as we are also involved in the patients' treatment groups, as discussed with examples in the response paper to Weegmann and Head (Cilasun, 2016). Staff manage the confidentiality for both the patient and the family member, for example we may facilitate the Families and Friends Group, as well as the treatment group that their loved one attends. There are two members of staff facilitating the group, one a Consultant Psychiatrist and the other a Mental Health Nurse both trained in MBT. We occasionally have students or other staff as observers.

The detailed structure of each session is as follows:

10 minutes Arrival, introductions, a sense of what each member wants to get out of the meeting

15 minutes Presentation on a topic, for example 'Mentalization - what is it, how does break down?' followed by questions and discussion

50 minutes Role-play or exercises in dyads, triads or as a group. The content of the exercises or role-play is related to issues group members have brought in the context of the topic

10 minutes A review of the meeting and identifying content for the next meeting 
Topics for each session are as follows:

Topic Covered in a rolling manner

\begin{tabular}{|l|l|}
\hline Empathic Validation & Mindfulness - what it is, basic practice \\
and how it relates to mentalization
\end{tabular}

Table 1

The Families and Friends Group mirrors the MBT treatment, with the psychoeducational content very similar to that in the Introductory Group of the MBT program. The skill building exercises which then follow, have the potential to increase the therapeutic impact of MBT for the patients of the service. 
As the group has progressed and developed, the psycho-educational element has enabled the group members to see how the mentalizing concept helps to understand the relationship and communication difficulties with their loved one. For example a mother commented:

'I used to tell my daughter to stop being so disrespectful and not to talk to me that way. Now I can recognise that neither of us were mentalizing and my response was only making the situation worse.'

We teach group members firstly to attune to and mentalize themselves before responding to their loved one with empathy. This is followed by mentalizing the 'other', that is attempting to understand what is in their loved one's mind. The last step is mentalizing with their loved one about the situation. This sequence in practice is fraught with pitfalls. We use role play, linking an example of an interaction they have brought, to the educational material in the session.

\section{Process of the Families and Friends Group}

The Families and Friends Group members feel supported by sharing their feelings and experiences with others. They have a sense of being understood when they hear others have similar experiences, similar pain and difficulties. A comment from a parent: 
'I feel almost a sense of relief that I'm not the only one trying to live with a daughter who has a personality disorder. I manage better for attending the group than I would have otherwise.'

Group members appreciate the practical exercises. They value the opportunity to learn and practice new skills, through role play. They recognise the problems and have the desire to change the patterns in their communication with their loved ones. Some support groups can perpetuate the patient as the problem whereas this way, the problem is seen to be clearly in the communication system. A mother wrote of the group:

'It has made me think more about how I interact with my daughter and made me more aware of trying to reflect back to her.'

Group members have reported this psycho-educational and skills building group is experienced as more supportive than traditional groups where people only share difficulties. It may well be that due to the structure that is provided, it is easier to share difficulties with others. In comparison to other support groups, which they also use, one difference which they value with this group, is the professional input of the teaching from the clinicians. 
The group members have also brought information about talks, webinars, books, or other support groups available. Some group members have at times met for dinner following the group. The group climate is compassionate, the emotional tone of the meetings is warm and supportive. Members actively advise each other on self-care and a supportive atmosphere emphasizes not having to manage alone. The most poignant groups have worked with grief of the loss in its several different forms, loss of health, career prospects and a 'normal' family life. The group members have spoken about the shame with the diagnosis of borderline personality disorder and the impact it has on the relationship with their extended family and friends who struggle to understand the person's behaviour. This often leads to isolation for the whole family. Sharing with others who understand in this group starts the process of being able to talk, thus reducing shame, stigma and social isolation.

The group has heard from carers who are being abused verbally and accused of being the aggressor. Others have expressed a painful estrangement from their family member feeling hopeless as to how to re-establish communication. Although it is not possible to immediately change this situation, encouraging the family member to take care of their own wellbeing and try different strategies to communicate enables them to manage the situation more effectively. Equally elderly parents who have been full time at hand to manage suicidal crises were able to go on holiday for the first time in many years as their adult offspring improved in the treatment program. 


\section{Evaluation}

We conducted a formal evaluation of the group between November 2016 and January 2017 collecting information using a questionnaire with quantitative and qualitative questions which we designed. We supplemented this information from our own records of attendance. The group had been running for three years having started in November 2013. We identified that the group members have been attending for between one and three years, attending between $1-18$ sessions in that time. A total of 18 people have attended over the life of the group. This evaluation did not catch those who attended only once, as during the evaluation period we did not have any new attendees. The majority of group members have been female with adult children who have been diagnosed with BPD, however there have also been two fathers in the group who have attended regularly. Other group members have partners who have been diagnosed with BPD. The ethnic and cultural mix of the group has been multicultural with a mix of British, European and Global first generation migrants, with these families settled in the borough of London we serve.

For the evaluation, the group was asked to complete a questionnaire which included quantitative evaluation of learning using a Likert Scale (1932), followed by four questions to obtain qualitative data on the group experience. 
Table 2

Quantitative Evaluation of Learning

On a scale of 0 to 5 , with 0 being not at all useful to 5 being very useful, please tell us how useful the group has been for:

a. Learning about Emotionally Unstable or Borderline Personality Disorder and Mentalization Based Therapy approaches

b. Learning and practicing different styles of communication

c. Supporting by sharing experiences with others

This was followed by qualitative questions, which were open aimed at finding out more about the experience and usefulness of the groups. The questions are in table 3 .

Table 3

Qualitative Questions

- Have you noticed any changes in how you have been managing since coming to this group?

- What elements of the group have you found most helpful?

- What would improve the Friends and Families group for you?

- Any other comments? 


\section{$\underline{\text { Results }}$}

A total of 11 questionnaires were send out and 7 were received back .

Learning evaluation

With the exception of one respondent who scored 4 for a and b, the rest of the respondents scored 5 in all areas.

Thematic analysis of the qualitative data

Educational aspects:

Learning about the disorder, mentalization, mindfulness, practising ways to communicate were listed as important. There was a request for information about current research into the disorder and treatments.

Support and guidance:

Shared experience was valued. There was gratitude expressed and a wish for more frequent meetings.

'Very helpful to know I can come to explain difficult situations and keep getting some guidance.' 
'Very supportive.' Has helped me to understand my daughter's needs and not to give up.'

Change in the carer and the relationships:

Group members reported improvements in their own mental health, less stress in their lives, and improved relationships, with some examples as below.

'I am able to think first instead of just reacting'.

'It has helped my own mental health and has created an avenue of discussion between me and my daughter.'

'Better way of communicating as parents and coordinating on communication with our daughter who has mental health issues.'

\section{Vignettes from groups}

We sought and obtained permission from the group members to write vignettes from the group sessions. These are shown below. The vignettes are altered to protect confidentiality, but they give examples of common themes. We shared the final paper with the group members.

“I blamed myself for my daughter's difficulties: maybe I didn't love her enough? Maybe I didn't show her enough care? The non-judgmental 
atmosphere in the group, the feeling that we are in this together and the trust that everything that is discussed within the group is confidential has helped me to open up. Coming to the sessions has helped heal my guilt over time and I have stopped blaming myself. This has helped me feel happy within myself. This has helped me become more outwardly-looking which in turn has helped me better in my relationship with my daughter.”

Another mother described how rejected she feels when her daughter does not want to talk to her.

'Since coming here I see it is not anything I have done. It still hurts when she leaves the room but I don't blame myself now, try not to, although it is difficult.

In both of these examples the work of the group was to understand the mother's point of view and how in the present situation the guilt from the past is not helpful. The mothers are then able to be emotionally available for their daughters in the current situation.

Another mother reflected on the effect of their acrimonious divorce on her daughter. 'She blames me like her father does and doesn't talk to me.' 
In these cases it seemed important to understand the situations and work to move from blaming to understanding. The blaming dynamic is particularly common and strong, in the families group the mother typically taking on the blame. It is the same dynamic as in their daughters, the identified patients, in the form of self-blame, and then often self-attack, under the pressure of wanting to succeed and seeing their inability to have successful relationships. The group found a shared theme of siblings who got married or were able to lead a 'successful life' with a career as being a trigger to the patient's distress. If we move beyond blame then we find deep sadness and grief and compassion for the inability to manage to achieve and for the striving that causes so much hurt.

The following discussion occurred between two family group members, a younger woman who is the wife of a patient (A) and the other the father of a young woman (B).

A. You are the same age as my father, and he would never talk about these things, I have a brother who has mental health issues but they would never acknowledge it.

B. I was like that, like your father, that was me!

A. But you are here, you changed 
B. I love my child, I had no choice, I have a beautiful daughter, I had to learn...

This father had said earlier in the same group to the wife of a patient,

'I know what is like to be redundant, to lose one's self-worth after redundancy', helping understand and mentalise the situation for this other family, when the husband lost his job due to redundancy. He went on to explain how his experience of redundancy had increased his empathy for his daughter, with her difficulties.

\section{Discussion}

This evaluation has shown that the experience of the group is a highly positive one. This experience is of course limited by the small number of participants in the group and our lack of information about those who no longer attend.

It has been shown that the group leads to understanding situations in a different way, not just as an illness or a failure of their loved one, but a state which has developed as a result of factors which affects the whole family and has led to the particular presentation of disorder. Mentalizing the context, or in other words, the developing of a narrative or an understanding for the family, is providing a container and an 
antidote for the non-mentalising states of borderline personality disorder. Thomas Fuchs (2007) proposes that increasing prevalence of borderline personality disorder is linked to the development of mainly externally driven, fragmented character in postmodern society. Groups with their inherent potential for making connections, and group analytic framework in particular, are the ideal mode of intervention for the distress of the BPD in families.

We see the Families and Friends group as an application of group analysis. By application we mean the group is not an analytic group; the individual and group defenses are not articulated or analysed in the group. The frequency of the groups being monthly and the structured nature of sessions also mark this group as not being group analysis. But the use of the group process for support and change, and the empowerment of the members of the group to be each other's source of help are the application of group-analytic principles. The training aspect of the group takes the ‘ego training in action’ which Foulkes (Foulkes,1964: 82) named, a step forward, by including a formal training part of the group.

There are differences and similarities between therapy groups, support groups and psycho-educational groups. The MBT introductory group is an educational group which some patients find transformative in our experience. Some therapy groups, aimed to be transformative may not be experienced as supportive, especially during times of conflict. On the other hand traditional support groups may focus more on 
information giving and support for carers rather than seeking for change to occur. All these activities take place using group interaction. This group reports change in the members, despite the individual and group defenses not being worked with in the traditional analytic sense. The most surprising aspect of this group is that it leads to changes in people and in relationships despite being a once a month drop-in psychosocial intervention and not a therapy group. This may be because it utilises the natural commitment the family members have to their loved ones to change the emotional environment of their situations.

We started with the question, 'How might we offer something of use to families and carers of the patients we treat?' A group that provides information, gives opportunity to learn and practice communication styles and encourage these group members to recognize when they are not able to mentalize, seems to be helpful and reduces stress in the families. The sharing of experiences and learning in this way with other families is experienced as supportive. Improvement of family members’ mental health has been commented on in the evaluations, and it is certainly our impression that the attendees continue to attend because they find the group helpful for their own wellbeing.

We feel that more can be done with a systemic approach with this treatment population, prioritising the family unit for treatment rather than the individual right from the start. An example of this is the open dialogue approach where there is an 
emphasis on creating dialogue within the treatment system and families (Olson, Seikkula and Ziedonis, 2014). If the family is the unit of treatment, the considerations around confidentiality dissolve.

Acknowledgments; gratitude for the stimulating discussions and support of colleagues, Martin Weegmann, Ryan Kemp, Monica Doran, John Schalpobersky, Lawrence Ladden, Diana Menzies and Xenya Kantaris. 


\section{References}

Bateman A and Fonagy P (2006) Mentalization-Based Treatment for borderline personality disorder. Oxford: Oxford University Press.

Bateman A and Fonagy P (2012) Handbook of Mentalizing in Mental Health Practice. American Psychiatric Association.

Burgess R C (2007) ‘A Model for Enhancing Individual and Organisational Learning of 'Emotional Intelligence’: The Drama and Winner's Triangles’. Social Work Education 24 (1), pp. 97-112. Available at: doi.org/10.1080/0261547052000325008 (Accessed:11 January 2018)

Cilasun J (2016) 'Circles of Care and Ties that Bind-Response to Weegmann and Head’. Group Analysis 49(4), pp. 452 - 456.

Foulkes SH (1964) Therapeutic Group Analysis. London: George Allen \& Unwin Ltd.

Fuchs T (2007)' Fragmented Selves: Temporality and Identity in Borderline Personality Disorder’. Psychopathology. 40 (6), pp. 379-387. 
Great Britain. NHS England (2017). The Long Term Conditions: Year of Care Commissioning Programme Implementation Handbook. Available at: https://www.england.nhs.uk/wp-content/uploads/2017/02/ltc-yoc-handbook.pdf (Accessed: 18 January 2018)

Great Britain. National Institute for Clinical Excellence (2009) Borderline personality disorder: Treatment and Management [Online]. Available at: http://publications.nice.org.uk/borderline-personality-disorder-cg78 (Accessed: 18 January 2018)

Great Britain. National Institute for Mental Health in England (2003) Breaking the cycle of rejection: The personality disorder capabilities framework [Online]. Available at: http://www.spn.org.uk/fileadmin/spn/user/*.pdf/Papers/personalitydisorders.pdf. (Accessed: 18 January 2018).

Hoffman, P.D., Fruzzetti, A. E. \& Buteau, E. (2007) ‘Understanding and engaging families: An Education, Skills and Support Program for relatives impacted by Borderline Personality Disorder’ Journal of Mental Health 16 (1), pp.69-82 
Likert, R. (1932). 'A Technique for the Measurement of Attitudes'. Archives of Psychology. 140, pp. 1-55.

NHS England Commissioning for Carers Principles Available at:

https://www.england.nhs.uk/commissioning/comm-carers/principles/ (Accessed: 24 January 2018).

Olson, M., Seikkula, J., Ziedonis, D. (2014) The key elements of dialogic practice in open dialogue: Fidelity Criteria. Available at:

https://www.umassmed.edu/globalassets/psychiatry/opendialogue/keyelementsv1.109022014.pdf (Accessed: 5 January 2018).

Porr, V. (2010) Overcoming borderline personality disorder: A family guide for healing and change. New York: Oxford University Press.

Rethink (2003) Under pressure Available at: www.rethink.org/publications. (Accessed: 11 January 2018).

Weegmann, M., Head, A. (2016) 'Circles of Care: Applied Group Analysis with Family and Friends', Group Analysis 49(4): pp. 431-452. 